EPJ manuscript No.

(will be inserted by the editor)

\title{
Modulational instability, rogue waves, and envelope solitons in opposite polarity dusty plasmas
}

\author{
M. H. Rahman*, N. A. Chowdhury, A. Mannan, M. Rahman, and A. A. Mamun \\ Department of Physics, Jahangirnagar University, Savar, Dhaka-1342, Bangladesh \\ E-mail: rahman1992phy@gmail.com*
}

Received: date / Revised version: date

\begin{abstract}
Dust-acoustic (DA) waves (DAWs) and their modulational instability (MI) have been investigated theoretically in a plasma system consisting of inertial opposite polarity (positively and negatively) warm adiabatic charged dust particles as well as inertialess non-extensive $q$-distributed electrons and nonthermal ions. A nonlinear Schrödinger (NLS) equation is derived by using the reductive perturbation method. It has been observed from the analysis of NLS equaion that the modulationally stable solitary DAWs give rise to the existence of dark envelope solitons, and that the modulationally unstable solitary DAWs give rise to the existence of bright envelope solitons or rogue structures. It is also observed for the fast mode of DAWs that the basic features (viz. stability of the DAWs, MI growth rate, amplitude and width of the DA rogue waves, etc.) are significantly modified by the related plasma parameters (viz. dust masses, dust charge state, non-extensive parameter $q$, and non-thermal parameter $\alpha$ ). The results of our present investigation might be useful for understanding different nonlinear electrostatic phenomena in both space (viz. ionosphere and mesosphere) and laboratory plasmas (viz. high intensity laser irradiation and hot cathode discharge).
\end{abstract}

PACS. PACS-key discribing text of that key - PACS-key discribing text of that key

\section{Introduction}

Recently, the research regarding dusty plasma is one of the fundamental and exponentially growing branches of plasma physics because of the empirical results directly support the existence of dust not only in space plasmas (viz. cometary tails, asteroid zones, planetary ring, interstellar medium, lower part of the earths ionosphere, and the magnetosphere, etc.) but also in laboratory plasmas (viz. radio frequency plasma discharge, low temperature physics, and plasma crystals, etc [1,2, 3, 4,5,6].). Dust acoustic (DA) waves (DAWs) and their associated nonlinear structures (viz. shock, vortices, rogue, and envelope solitons, etc.) are rigorously used by Plasma physicists to understand the collective behaviours of such kind of dusty plasmas (DP). Usually, dust grains are assumed negatively charged massive objects due to the collection of electrons from background of plasma species [7, 8. But a set of policies (e.g. photoemission under ultraviolet radiation or thermionic emission from grains heated by radiative sources) by which a dust particle can acquire positive charge, and also lived along with negatively charged dust particles, ions, and electrons in various DP (viz. upper mesosphere, cometary tails, and Jupiters magnetosphere 9, 10, 11, etc.).

Maxwellian distribution, which was developed by Maxwell and Boltzmann, is suitable to describe the motion of particles in a thermodynamically equilibrium system. But in the astrophysical objects and space plasmas due to the various physical mechanisms (viz. wave-particle interaction, particle-particle interaction, and presence of external force field in natural space plasma environment), Maxwellian distribution is not adequate to describe the motion of plasma particles. A number of authors have used non-extensive $q$-distribution [12,13, 14, 15, or non-thermal Cairn's distribution 16, 17, 18 instead of Maxwellian distribution to describe the motion of plasma particles in thermodynamically nonequilibrium system. Rogue waves (RWs), which generate due to the modulational instability (MI) of plane water waves, observed first in ocean [19]. Nowadays, RWs can be observed in optics, atmospheric physics, stock market crashes [20, super-fluid helium [21], and in plasma physics [22, etc. Sayed and Mamun [23] examined the presence of positive dust component significantly modify the fundamental properties of solitary potential structures along with negative dust in four component DP. El-Taibany 24] investigated the dependency of DA solitary waves nature on opposite charge polarity dust grain masses and temperature. Bains et al. [25] reported DAWs modulation in the presence of $q$-distributed electrons and ions and found that non-extensive ions have more effects on the MI of the DAWs than 
electrons. Misra and Chowdhury [26] studied MI of DAWs in a DP with non-thermal electrons and ions. Zaghbeer et al. [27. examined the effects of $q$-distributed electrons and ions on DA RWs (DARWs) in opposite polarity DP. Moslem et al. 28 investigated the DARWs in a $q$-distributed plasma and found that RWs are influenced by the plasma parameters. Sultana et al. 29] studied envelope solitons and their MI in DP and found that the MI conditions of the modified envelope solitons are also influenced due to the variation of the intrinsic plasma parameters. Therefore, in our present work, we will examine the MI of the DAWs propagating in opposite polarity DP (inertial warm negatively and positively charged dust particles) as well as inertialess $q$-distributed electrons and non-thermal ions which abundantly occurs in astrophysical environments, (viz. upper mesosphere, cometary tails, and Jupiters magnetosphere, etc).

The present paper is organized as follows: In Sec. 2 the model equations and derivation of the NLS equation

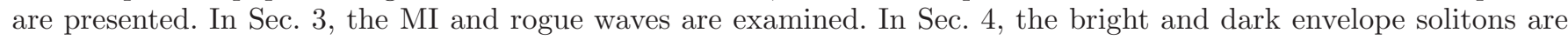
observed. In sec. [5 a brief discussion is provided.

\section{Model equations and derivation of the NLS equation}

We consider a collisionless, fully ionized, unmagnetized plasma system comprising of inertial warm negatively charged dust particles (mass $m_{1}$; charge $q_{1}=-Z_{1} e$ ), and positively charged dust particles (mass $m_{2}$; charge $q_{2}=+Z_{2} e$ ), as well as $q$-distributed electrons (mass $m_{e}$; charge $-e$ ), and non-thermal ions (mass $m_{i}$; charge $+e$ ). $Z_{1}\left(Z_{2}\right)$ is the number of electrons (protons) residing on a negative (positive) dust particle. At equilibrium, the quasi-neutrality condition can be expressed as $Z_{1} n_{10}+n_{e 0}=Z_{2} n_{20}+n_{i 0}$; where $n_{10}, n_{e 0}, n_{20}$, and $n_{i 0}$ are the equilibrium number densities of warm negatively charged dust particles, $q$-distributed electrons, positively charged dust particles, and non-thermal ions, respectively. The normalized governing equations of the DAWs in our plasma system are

$$
\begin{aligned}
& \frac{\partial n_{1}}{\partial t}+\frac{\partial}{\partial x}\left(n_{1} u_{1}\right)=0, \\
& \frac{\partial u_{1}}{\partial t}+u_{1} \frac{\partial u_{1}}{\partial x}+3 \sigma_{1} n_{1} \frac{\partial n_{1}}{\partial x}=\frac{\partial \phi}{\partial x}, \\
& \frac{\partial n_{2}}{\partial t}+\frac{\partial}{\partial x}\left(n_{2} u_{2}\right)=0, \\
& \frac{\partial u_{2}}{\partial t}+u_{2} \frac{\partial u_{2}}{\partial x}+3 \sigma_{2} n_{2} \frac{\partial n_{2}}{\partial x}=-a \frac{\partial \phi}{\partial x}, \\
& \frac{\partial^{2} \phi}{\partial x^{2}}=\left(\mu_{i}+b-1\right) n_{e}-\mu_{i} n_{i}+n_{1}-b n_{2},
\end{aligned}
$$

where $n_{1}\left(n_{2}\right)$ is the number density of negatively (positively) charged dust particles normalized by its equilibrium value $n_{10}\left(n_{20}\right) ; u_{1}\left(u_{2}\right)$ is the negatively (positively) charged dust fluid speed normalized by $C_{1}=\left(Z_{1} T_{i} / m_{1}\right)^{1 / 2}$, and the electrostatic wave potential $\phi$ is normalized by $T_{i} / e$ (with $e$ being the magnitude of an electron charge); $T_{1}, T_{2}, T_{i}$, and $T_{e}$ is the temperature of negatively charged dust, positively charged dust, non-thermal ions, and $q-$ distributed electrons, respectively; the time and space variables are normalized by $\omega_{p d 1}^{-1}=\left(m_{1} / 4 \pi Z_{1}^{2} e^{2} n_{10}\right)^{1 / 2}$ and $\lambda_{D d 1}=\left(T_{i} / 4 \pi Z_{1} e^{2} n_{10}\right)^{1 / 2}$, respectively; some related parameters are defined as $a=m_{1} Z_{2} / m_{2} Z_{1}, b=Z_{2} n_{20} / Z_{1} n_{10}$ $\mu_{i}=n_{i 0} / Z_{1} n_{10}, \sigma_{1}=T_{1} / Z_{1} T_{i}$, and $\sigma_{2}=T_{2} m_{1} / Z_{1} T_{i} m_{2}$. The expression for the number density of non-extensive electrons following the non-extensive $q$-distribution [12,13, 14, can be written as

$$
n_{e}=[1+(q-1) \delta]^{\frac{q+1}{2(q-1)}}
$$

where $\delta=T_{i} / T_{e}$ and $q$ is the non-extensive parameter. When $q<1(q>1)$ refers to super-extensivity (sub-extensivity) [15] and $q=1$ refers to Maxwellian [4]. The expression for the number density of non-thermal ions following the nonthermal Cairn's distribution [16]17, 18, can be written as

$$
n_{i}=\left(1+\beta \phi+\beta \phi^{2}\right) \exp (-\phi)
$$

where $\beta=4 \alpha /(1+3 \alpha)$ with $\alpha$ being the non-thermal parameter. We note that in many space plasma systems contain fraction of energetic or fast plasma particle in addition to thermal ones. Now, by substituting Eqs. (6) and (7) into Eq. (5), and expanding up to third order in $\phi$, we get

$$
\frac{\partial^{2} \phi}{\partial x^{2}}=b-1+n_{1}-b n_{2}+\gamma_{1} \phi+\gamma_{2} \phi^{2}+\gamma_{3} \phi^{3}+\cdots \cdot
$$


where

$$
\begin{aligned}
\gamma_{1} & =\frac{1}{2}\left[\left(b+\mu_{i}-1\right)(q+1) \delta+2 \mu_{i}(1-\beta)\right], \\
\gamma_{2} & =\frac{1}{8}\left[\left(b+\mu_{i}-1\right)(q+1)(3-q) \delta^{2}-4 \mu_{i}\right], \\
\gamma_{3} & =\frac{1}{48}\left[\left(b+\mu_{i}-1\right)(q+1)(q-3)(3 q-5) \delta^{3}+8 \mu_{i}(1+3 \beta)\right] .
\end{aligned}
$$

To study the MI of the DAWs, we will derive the NLS equation by employing the reductive perturbation method. So, we first introduce the stretched co-ordinates

$$
\begin{aligned}
& \xi=\epsilon\left(x-v_{g} t\right), \\
& \tau=\epsilon^{2} t
\end{aligned}
$$

where $v_{g}$ is the envelope group velocity and $\epsilon(0<\epsilon<1)$ is a small (real) parameter. Then, we can write a general expression for the dependent variables as

$$
G(x, t)=G_{0}+\sum_{m=1}^{\infty} \epsilon^{(m)} \sum_{l=-\infty}^{\infty} G_{l}^{(m)}(\xi, \tau) \exp (i l \Upsilon)
$$

where $G_{l}^{(m)}=\left[n_{1 l}^{(m)}, u_{1 l}^{(m)}, n_{2 l}^{(m)}, u_{2 l}^{(m)}, \phi_{l}^{(m)}\right]^{T}, G_{0}=[1,0,1,0,0]^{T}, \Upsilon=(k x-\omega t)$, and $k(\omega)$ is the fundamental carrier wave number (frequency). All elements of $G_{l}^{(m)}$ satisfy the reality condition $G_{-l}^{(m)}=G_{l}^{*(m)}$, where the asterisk indicates the complex conjugate. The derivative operators in the above equations are treated as follows:

$$
\begin{aligned}
& \frac{\partial}{\partial t} \rightarrow \frac{\partial}{\partial t}-\epsilon v_{g} \frac{\partial}{\partial \xi}+\epsilon^{2} \frac{\partial}{\partial \tau} \\
& \frac{\partial}{\partial x} \rightarrow \frac{\partial}{\partial x}+\epsilon \frac{\partial}{\partial \xi} .
\end{aligned}
$$

Now, by substituting Eqs. (9)-(13) into Eqs. (19)-(41), and (8), and equating the coefficients of $\epsilon$ for $m=l=1$, we obtain

$$
\begin{aligned}
n_{11}^{(1)} & =\frac{k^{2}}{S} \phi_{1}^{(1)}, & u_{11}^{(1)} & =\frac{k \omega}{S} \phi_{1}^{(1)}, \\
n_{21}^{(1)} & =\frac{a k^{2}}{A} \phi_{1}^{(1)}, & u_{21}^{(1)} & =\frac{a k \omega}{A} \phi_{1}^{(1)},
\end{aligned}
$$

where $S=\lambda k^{2}-\omega^{2}, A=\omega^{2}-\theta k^{2}, \lambda=3 \sigma_{1}$, and $\theta=3 \sigma_{2}$. We thus obtain the dispersion relation for DAWs

$$
\omega^{2}=\frac{k^{2} M \pm k^{2} \sqrt{M^{2}-4 G H}}{2 G},
$$

where $M=\theta k^{2}+\lambda k^{2}+\theta \gamma_{1}+\lambda \gamma_{1}+a b+1, G=k^{2}+\gamma_{1}$, and $H=\theta \lambda k^{2}+\theta \gamma_{1} \lambda+\theta+a b \lambda$. To obtain real and positive values of $\omega$ from Eq. (15), the condition $M^{2}>4 G H$ should be verified. The positive sign in Eq. (15) corresponds to the fast DA mode $\left(\omega_{f}\right)$, whereas the negative sign corresponds to the slow DA mode $\left(\omega_{s}\right)$. Physically, in fast mode both dust species oscillate in same phase with electrons and ions. On the other hand, in slow mode one of the dust species oscillates in opposite phase with electrons, ions, and another dust species. We have numerically analyzed the $\omega_{f}$ and $\omega_{s}$ in Figs. 1 and 2, which clearly indicate that (a) as we increase the value of carrier wave number $k$, firstly, the $\omega_{f}$ increases exponentially but after a particular value of $k, \omega_{f}$ remains almost constant (please see Fig. 1); (b) the $\omega_{f}$ increases with the increase of $Z_{2}$ for fixed value of $n_{20}, Z_{1}$, and $n_{10}$ (via $\left.b=Z_{2} n_{20} / Z_{1} n_{10}\right)$; (c) as we increase the value of $k$, the $\omega_{s}$ linearly increases (please see Fig. 2); (d) the $\omega_{s}$ increases with the increase of $Z_{1}$ for fixed value of $Z_{2}, n_{20}$, and $n_{10}$ (via $\left.b=Z_{2} n_{20} / Z_{1} n_{10}\right)$. So, the charge of the dust particles play an opposite role for the $\omega_{f}$ and $\omega_{s}$ of the DAWs, respectively. The second-order of $\epsilon$ when $(m=2)$ reduced equations with $(l=1)$ are

$$
\begin{aligned}
& n_{11}^{(2)}=\frac{k^{2}}{S} \phi_{1}^{(2)}+\frac{i}{S^{2}}\left(\lambda k^{3}+k \omega^{2}-2 v_{g} \omega k^{2}-k S\right) \frac{\partial \phi_{1}^{(1)}}{\partial \xi} \\
& u_{11}^{(2)}=\frac{k \omega}{S} \phi_{1}^{(2)}+\frac{i}{S^{2}}\left(\lambda \omega k^{2}+\omega^{3}-2 v_{g} k \omega^{2}-v_{g} k S\right) \frac{\partial \phi_{1}^{(1)}}{\partial \xi},
\end{aligned}
$$




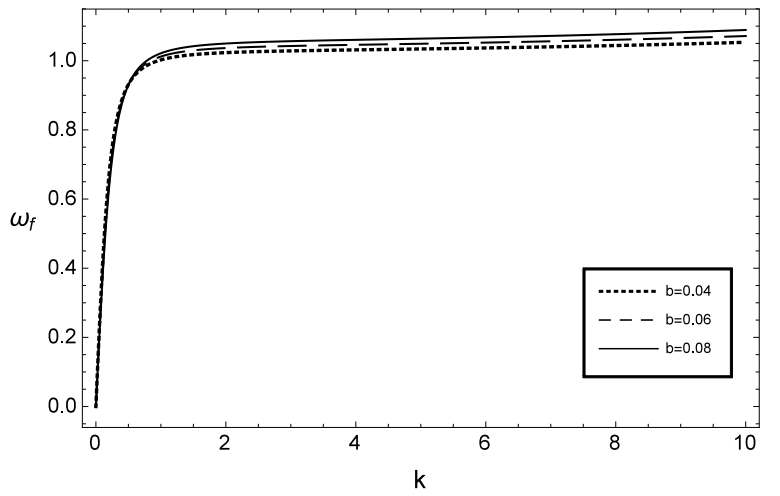

Fig. 1. The variation of $\omega_{f}$ with $k$ for different values of $b$; along with $a=1.5, q=1.3, \alpha=0.3, \delta=0.4, \mu_{i}=0.6, \sigma_{1}=0.0001$, and $\sigma_{2}=0.001$.

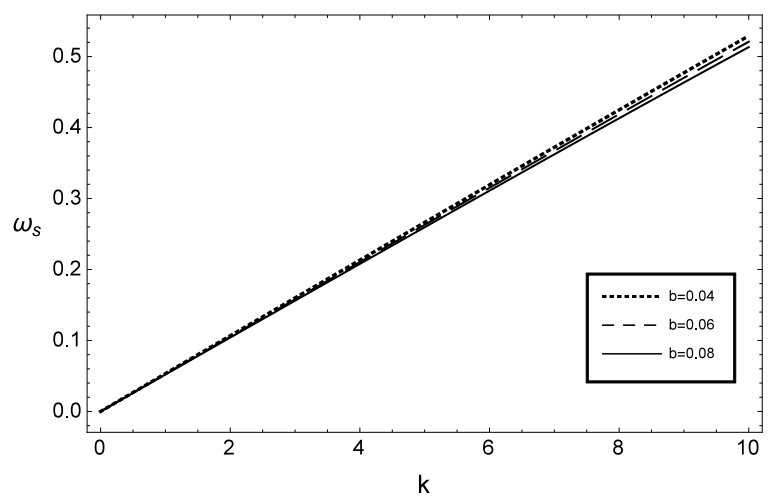

Fig. 2. The variation of $\omega_{s}$ with $k$ for different values of $b$; along with $a=1.5, q=1.3, \alpha=0.3, \delta=0.4, \mu_{i}=0.6, \sigma_{1}=0.0001$, and $\sigma_{2}=0.001$.

$$
\begin{aligned}
& n_{21}^{(2)}=\frac{a k^{2}}{A} \phi_{1}^{(2)}-\frac{i a}{A^{2}}\left(\theta k^{3}+k \omega^{2}+k A-2 \omega v_{g} k^{2}\right) \frac{\partial \phi_{1}^{(1)}}{\partial \xi}, \\
& u_{21}^{(2)}=\frac{a k \omega}{A} \phi_{1}^{(2)}-\frac{i a}{A^{2}}\left(\theta \omega k^{2}+\omega^{3}+v_{g} k A-2 v_{g} k \omega^{2}\right) \frac{\partial \phi_{1}^{(1)}}{\partial \xi},
\end{aligned}
$$

with the compatibility condition

$$
v_{g}=\frac{F 1-2 S^{2} A^{2}-S A(A-a b S)}{2 k \omega\left(A^{2}+a b S^{2}\right)},
$$

where

$$
F 1=k^{2}\left(\lambda A^{2}+a b \theta S^{2}\right)+\omega^{2}\left(A^{2}+a b S^{2}\right) .
$$

The second-order of $\epsilon$ when $(m=2)$ reduced equations with $(l=1)$ are

$$
\begin{array}{ll}
n_{12}^{(2)}=C_{1}\left|\phi_{1}^{(1)}\right|^{2}, & n_{10}^{(2)}=C_{6}\left|\phi_{1}^{(1)}\right|^{2}, \\
u_{12}^{(2)}=C_{2}\left|\phi_{1}^{(1)}\right|^{2}, & u_{10}^{(2)}=C_{7}\left|\phi_{1}^{(1)}\right|^{2}, \\
n_{22}^{(2)}=C_{3}\left|\phi_{1}^{(1)}\right|^{2}, & n_{20}^{(2)}=C_{8}\left|\phi_{1}^{(1)}\right|^{2}, \\
u_{22}^{(2)}=C_{4}\left|\phi_{1}^{(1)}\right|^{2}, & u_{20}^{(2)}=C_{9}\left|\phi_{1}^{(1)}\right|^{2}, \\
\phi_{2}^{(2)}=C_{5}\left|\phi_{1}^{(1)}\right|^{2} & \phi_{0}^{(2)}=C_{10}\left|\phi_{1}^{(1)}\right|^{2},
\end{array}
$$


where

$$
\begin{aligned}
C_{1}= & \frac{2 C_{5} k^{2} S^{2}-\left(3 \omega^{2} k^{4}+\lambda k^{6}\right)}{2 S^{3}}, \\
C_{2}= & \frac{\omega C_{1} S^{2}-\omega k^{4}}{k S^{2}}, \\
C_{3}= & \frac{3 a^{2} \omega^{2} k^{4}+\theta a^{2} k^{6}+2 a C_{5} A^{2} k^{2}}{2 A^{3}}, \\
C_{4}= & \frac{\omega C_{3} A^{2}-\omega a^{2} k^{4}}{k A^{2}}, \\
C_{5}= & \frac{F^{2}+b S^{3}\left(3 a^{2} \omega^{2} k^{4}+\theta a^{2} k^{6}\right)}{2 S^{2} k^{2} A^{3}+2 A^{3} S^{3}\left(4 k^{2}+\gamma_{1}\right)-2 a b A^{2} k^{2} S^{3}}, \\
F_{2}= & A^{3}\left(3 \omega^{2} k^{4}+\lambda k^{6}\right)-2 \gamma_{2} A^{3} S^{3}, \\
C_{6}= & \frac{2 v_{g} \omega k^{3}+\lambda k^{4}+k^{2} \omega^{2}-C_{10} S^{2}}{S^{2}\left(v_{g}^{2}-\lambda\right)}, \\
C_{7}= & \frac{v_{g} C_{6} S^{2}-2 \omega k^{3}}{S^{2}}, \\
C_{8}= & \frac{2 v_{g} \omega a^{2} k^{3}+\theta a^{2} k^{4}+a^{2} k^{2} \omega^{2}+a C_{10} A^{2}}{A^{2}\left(v_{g}^{2}-\theta\right)}, \\
C_{9}= & \frac{v_{g} C_{8} A^{2}-2 \omega a^{2} k^{3}}{A^{2}}, \\
C_{10}= & \frac{2 \gamma_{2} A^{2} S^{2}\left(v_{g}^{2}-\theta\right)\left(v_{g}^{2}-\lambda\right)+F 3}{a b A^{2} S^{2}\left(v_{g}^{2}-\lambda\right)+F 4}, \\
F_{3}= & A^{2}\left(2 v_{g} \omega k^{3}+\lambda S^{2}+k^{2} \omega^{2}\right)\left(v_{g}^{2}-\theta\right) \\
2 & \left.-v_{g}^{2} \omega a^{2} k^{3}+\theta a^{2} k^{4}+a^{2} k^{2} \omega^{2}\right)\left(v_{g}^{2}-\lambda\right), \\
2 & S^{2}\left(v_{g}^{2}-\theta\right)\left(v_{g}^{2}-\lambda\right) .
\end{aligned}
$$

Finally, the third harmonic modes $(m=3)$ and $(l=1)$ and with the help of Eqs. (14)-(17), give a system of equations, which can be reduced to the following NLS equation

$$
i \frac{\partial \Phi}{\partial \tau}+P \frac{\partial^{2} \Phi}{\partial \xi^{2}}+Q|\Phi|^{2} \Phi=0
$$

where $\Phi=\phi_{1}^{(1)}$ for simplicity. The dispersion coefficient $\mathrm{P}$ is given by

$$
P=\frac{F 5-A^{3} S^{3}}{2 A S \omega k^{2}\left(A^{2}+a b S^{2}\right)},
$$

where

$$
\begin{aligned}
F 5= & \left(v_{g} \omega A^{3}-\lambda k A^{3}\right)\left(\lambda k^{3}-2 \omega v_{g} k^{2}+k \omega^{2}-k S\right) \\
& +\left(v_{g} k A^{3}-\omega A^{3}\right)\left(\lambda \omega k^{2}-2 v_{g} k \omega^{2}+\omega^{3}-k v_{g} S\right) \\
& -a b S^{3}\left(v_{g} \omega-\theta k\right)\left(\theta k^{3}-2 \omega v_{g} k^{2}+k \omega^{2}+k A\right) \\
& -a b S^{3}\left(v_{g} k-\omega\right)\left(\theta \omega k^{2}-2 v_{g} k \omega^{2}+\omega^{3}+k v_{g} A\right) .
\end{aligned}
$$

The nonlinear coefficient $\mathrm{Q}$ is given by

$$
Q=\frac{A^{2} S^{2}\left\{2 \gamma_{2}\left(C_{5}+C_{10}\right)+3 \gamma_{3}\right\}-F 6}{2 \omega k^{2}\left(A^{2}+a b S^{2}\right)},
$$

where

$$
\begin{aligned}
F 6 & =2 \omega A^{2} k^{3}\left(C_{2}+C_{7}\right)+A^{2}\left(\omega^{2} k^{2}+\lambda k^{4}\right)\left(C_{1}+C_{6}\right) \\
& +2 a b \omega S^{2} k^{3}\left(C_{4}+C_{9}\right)+S^{2}\left(a b k^{2} \omega^{2}+a b \theta k^{4}\right)\left(C_{3}+C_{8}\right) .
\end{aligned}
$$




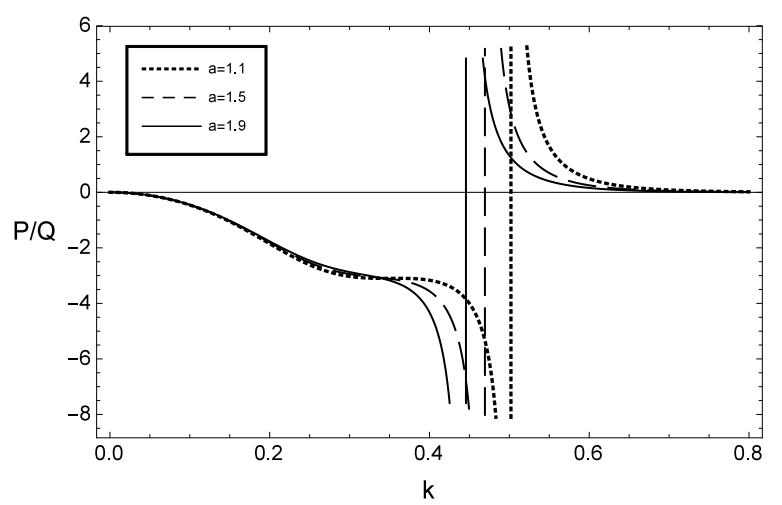

Fig. 3. The variation of $P / Q$ with $k$ for different values of $a$; along with $b=0.08, q=1.3, \alpha=0.3, \delta=0.4, \mu_{i}=0.6$, $\sigma_{1}=0.0001, \sigma_{2}=0.001$, and $\omega_{f}$.

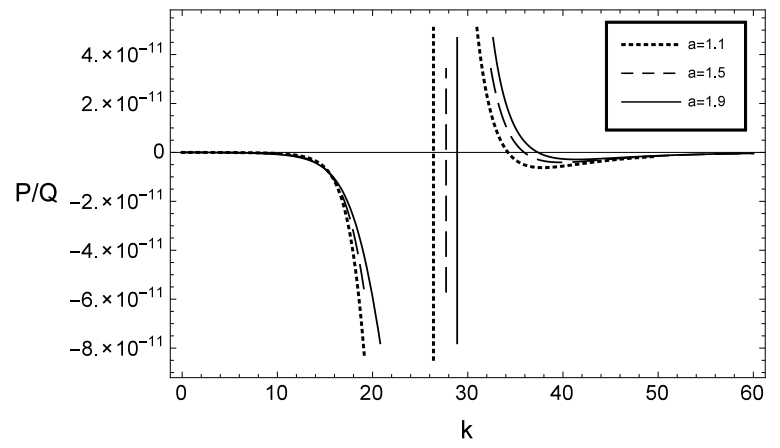

Fig. 4. The variation of $P / Q$ with $k$ for different values of $a$; along with $b=0.08, q=1.3, \alpha=0.3, \delta=0.4, \mu_{i}=0.6$, $\sigma_{1}=0.0001, \sigma_{2}=0.001$, and $\omega_{s}$.

\section{Modulational instability and rogue waves}

To study the MI of DAWs, we consider the linear solution of the NLS equation (18) in the form $\Phi=\hat{\Phi} e^{i Q|\hat{\Phi}|^{2} \tau}+c . c$ (c. c denotes the complex conjugate), where $\hat{\Phi}=\hat{\Phi}_{0}+\epsilon \hat{\Phi}_{1}$ and $\hat{\Phi}_{1}=\hat{\Phi}_{1,0} e^{i(\tilde{k} \xi-\tilde{\omega} \tau)}+$ c. c (the perturbed wave number $\tilde{k}$ and the frequency $\tilde{\omega}$ are different from $k$ and $\omega$ ). Now, by substituting these values in Eq. (18) the following nonlinear dispersion relation can be obtained as $[30,31,32,33,34$

$$
\tilde{\omega}^{2}=P^{2} \tilde{k}^{2}\left(\tilde{k}^{2}-\frac{2\left|\hat{\Phi}_{0}\right|^{2}}{P / Q}\right) .
$$

When $P / Q>0$, the DAWs are modulationally unstable against external perturbation, and in this region rogue waves and bright envelope solitons exist. On the other hand, when $P / Q<0$, the DAWs are modulationally stable and in this region dark envelope solitons exist. When $P / Q \rightarrow \pm \infty$, the corresponding value of $k\left(=k_{c}\right)$ is called critical or threshold wave number for the onset of MI. We have graphically shown how the ratio of $\mathrm{P} / \mathrm{Q}$ varies with $k$ for different values of $a$ in Fig. [3 and 4 , respectively. It is obvious from Fig. [3 and 4 that (a) for both $\omega_{f}$ and $\omega_{s}$, there is a stable/unstable region occurred for DAWs (see Fig. 3 and 4); (b) DAWs are modulationally stable (unstable) for long (short) wavelength; (c) the $k_{c}$ value decreases (increases), as we increase the value of $m_{1}\left(m_{2}\right)$ for fixed values of $Z_{2}$ and $Z_{1}$ (via $a=m_{1} Z_{2} / m_{2} Z_{1}$ ); (d) on the other hand, the $k_{c}$ increases (decreases), as we increase the value of $m_{1}\left(m_{2}\right)$ for fixed values of $Z_{2}$ and $Z_{1}$ (via $a=m_{1} Z_{2} / m_{2} Z_{1}$ ). When simultaneously $P / Q>0$ and $\tilde{k}<\tilde{k}_{c}=\left(2 P\left|\hat{\Phi}_{0}\right| / Q\right)^{1 / 2}$, 


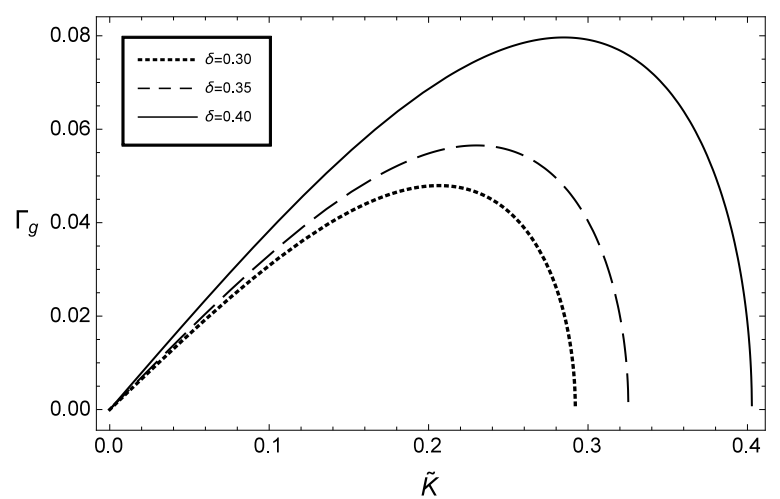

Fig. 5. Plot of the $\Gamma_{g}$ with $\tilde{k}$ for $\delta$; along with $k=0.5, \phi_{0}=0.5, a=1.5, b=0.08, q=1.3, \alpha=0.3, \mu_{i}=0.6, \sigma_{1}=0.0001$, $\sigma_{2}=0.001$, and $\omega_{f}$.

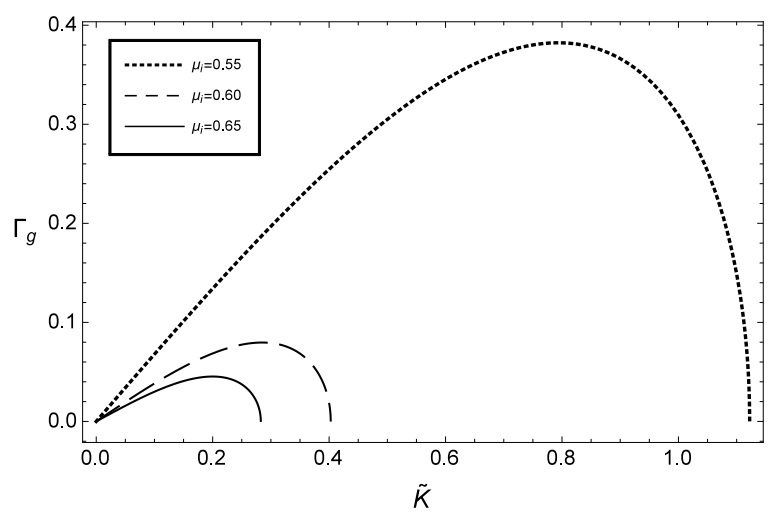

Fig. 6. Plot of the $\Gamma_{g}$ with $\tilde{k}$ for $\mu_{i}$; along with $k=0.5, \phi_{0}=0.5, a=1.5, b=0.08, q=1.3, \alpha=0.3, \delta=0.4, \sigma_{1}=0.0001$, $\sigma_{2}=0.001$, and $\omega_{f}$.

from Eq. (19) the growth rate $\left(\Gamma_{g}\right)$ of $\mathrm{MI}$ can be written as

$$
\Gamma_{g}=|P| \tilde{k}^{2}\left(\frac{\tilde{k}_{c}^{2}}{\tilde{k}^{2}}-1\right)^{1 / 2}
$$

We have graphically shown how the $\Gamma_{g}$ varies with $\tilde{k}$ for different values of $\delta, \mu_{i}$, and $\beta$ in Figs. 5- 7 . It is obvious from Figs. 5-77 that (a) the $\Gamma_{g}$ increases (decreases) with the increase of $T_{i}\left(T_{e}\right)$; (b) on the other hand, the $\Gamma_{g}$ increases with the decrease of $n_{i 0}$ for fixed value of $Z_{1}$ and $n_{10} ;$ (c) as we increases the value of $\beta$, the $\Gamma_{g}$ increases (see Fig. 7). The physics of this result is that, the maximum value of the growth rate of DAWs increases since the nonlinearity increases with the increase of the value of $\beta$. So, more non-thermal ions are used to enhance the maximum value of the growth rate. In the unstable region $(P / Q>0)$, the NLS equation (18) has rogue wave (rational) solution, which can be written as 35,36

$$
\Phi(\xi, \tau)=\sqrt{\frac{2 P}{Q}}\left[\frac{4(1+4 i P \tau)}{1+16 P^{2} \tau^{2}+4 \xi^{2}}-1\right] \exp (i 2 P \tau) .
$$

The solution (21) predicts the concentration of large amount of energy of the DAWs into a small, tiny region (please see Figs. 8 and 91) that is caused by the nonlinear behavior of the plasma medium. It can be seen from Fig. 8 and 9 that (a) as we increase the value of $q$ in sub-extensive (super-extensive) limit of the $q$, the amplitude and width of the rogue waves decrease (increase). Generally, when the nonlinearity of the plasma system increases, then excess nonlinearity leads to generate more energetic, taller rogue waves, by concentrating a reasonable amount of energy into tiny region. 


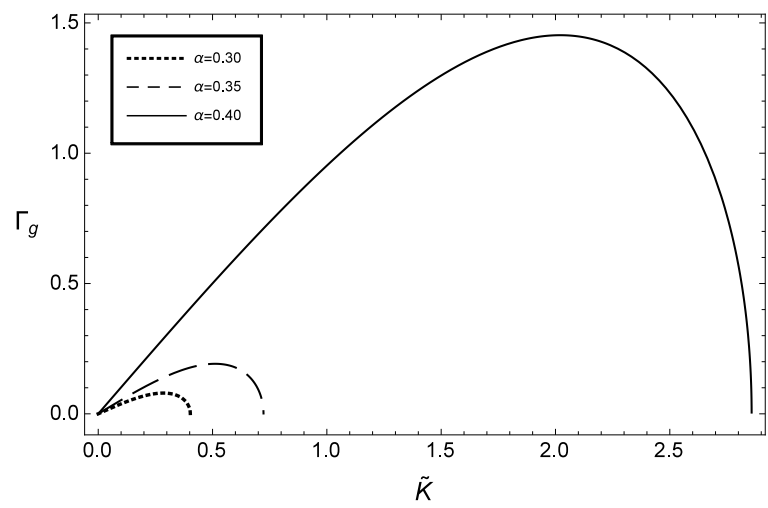

Fig. 7. Plot of the $\Gamma_{g}$ with $\tilde{k}$ for $\alpha$; along with $k=0.5, \phi_{0}=0.5, a=1.5, b=0.08, q=1.3, \delta=0.4, \mu_{i}=0.6, \sigma_{1}=0.0001$, $\sigma_{2}=0.001$, and $\omega_{f}$.

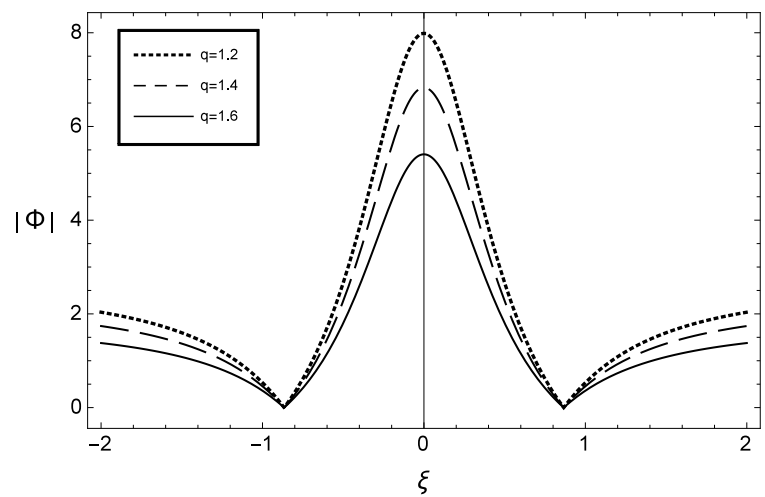

Fig. 8. The variation of $|\Phi|$ with $\xi$ for $\mathrm{q}=$ positive; along with $k=0.5, \tau=0, a=1.5, b=0.08, \alpha=0.3, \delta=0.4, \mu_{i}=0.6$, $\sigma_{1}=0.0001, \sigma_{2}=0.001$, and $\omega_{f}$.

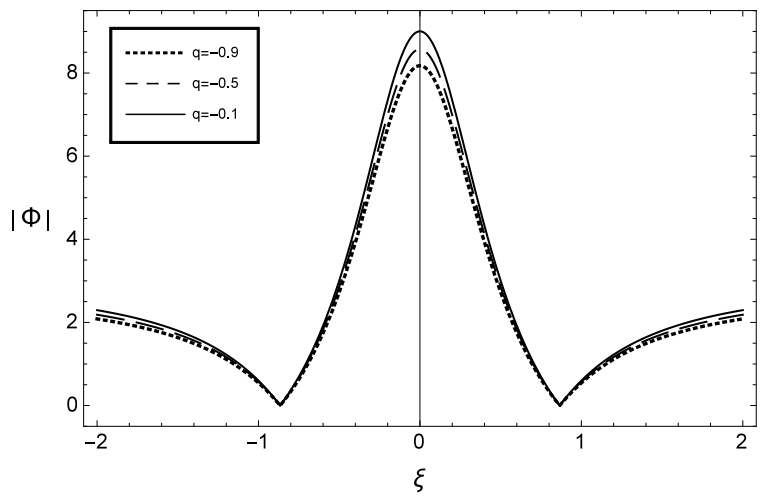

Fig. 9. The variation of $|\Phi|$ with $\xi$ for $\mathrm{q}=$ negative; along with $k=0.5, \tau=0, a=1.5, b=0.08, \alpha=0.3, \delta=0.4, \mu_{i}=0.6$, $\sigma_{1}=0.0001, \sigma_{2}=0.001$, and $\omega_{f}$. 


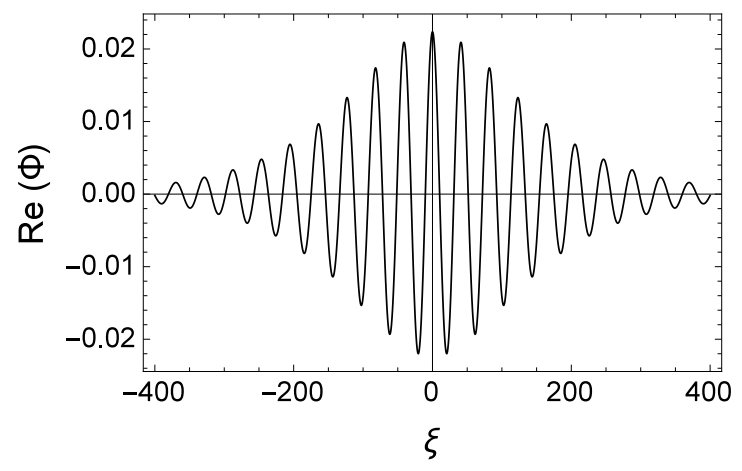

Fig. 10. The variation of $\operatorname{Re}(\Phi)$ with $\xi$ for bright envelope solitons; along with $k=0.5, \tau=0, \psi_{0}=0.0005, U=0.3, \Omega_{0}=0.4$, $a=1.5, b=0.08, q=1.3, \alpha=0.3, \delta=0.4, \mu_{i}=0.6, \sigma_{1}=0.0001, \sigma_{2}=0.001$, and $\omega_{f}$.

\section{Envelope solitons}

There are two types of envelope solitonic solutions exist, namely, bright and dark envelope solitons, depending on the sign of the coefficients $P$ and $Q$.

\subsection{Bright envelope solitons}

When $P / Q>0$, the expression of the bright envelope solitonic solution of Eq. (18) can be written in the given form [30, $31,32,33,34$

$$
\Phi(\xi, \tau)=\left[\psi_{0} \operatorname{sech}^{2}\left(\frac{\xi-U \tau}{W}\right)\right]^{1 / 2} \times \exp \left[\frac{i}{2 P}\left\{U \xi+\left(\Omega_{0}-\frac{U^{2}}{2}\right) \tau\right\}\right]
$$

where $\psi_{0}$ indicates the envelope amplitude, $U$ is the travelling speed of the localized pulse, $W$ is the pulse width, which can be written as $W=\left(2 P \psi_{0} / Q\right)^{1 / 2}$, and $\Omega_{0}$ is the oscillating frequency for $U=0$. The bright envelope soliton [obtained from Eq. (22)] is depicted in Fig. 10, It may be noted here the width of the bright envelope solitons decreases (increases) with the increase of $T_{i}\left(T_{e}\right)$ but their amplitude remains constant $\left(\right.$ via $\left.\delta=T_{i} / T_{e}\right)$.

\subsection{Dark envelope solitons}

As we know before that the condition for dark envelope soliton is $P / Q<0$. So, the dark envelope soliton solution of Eq. (18) can be written as [30, 31, 32, 33, 34,

$$
\Phi(\xi, \tau)=\left[\psi_{0} \tanh ^{2}\left(\frac{\xi-U \tau}{W}\right)\right]^{1 / 2} \times \exp \left[\frac{i}{2 P}\left\{U \xi-\left(\frac{U^{2}}{2}-2 P Q \psi_{0}\right) \tau\right\}\right] .
$$

The dark envelope soliton [obtained from Eq. (23)] is depicted in Fig. 11. It may be noted here the width of the dark envelope solitons increases (decreases) with the increase of $T_{i}\left(T_{e}\right)$ but their amplitude remains constant (via $\left.\delta=T_{i} / T_{e}\right)$.

\section{Discussion}

The amplitude modulation of DAWs structures has been theoretically investigated in an unmagnetized four component opposite polarity DP consisting of inertial warm positively and negatively charged dust particles as well as nonextensive electrons and non-thermal ions. A NLS equation, which governs the MI of DAWs and formation of associated rogue waves and bright envelope solitons in the unstable regimes, is derived by using reductive perturbation method. The results that have been found from our investigation can be summarized as follows 


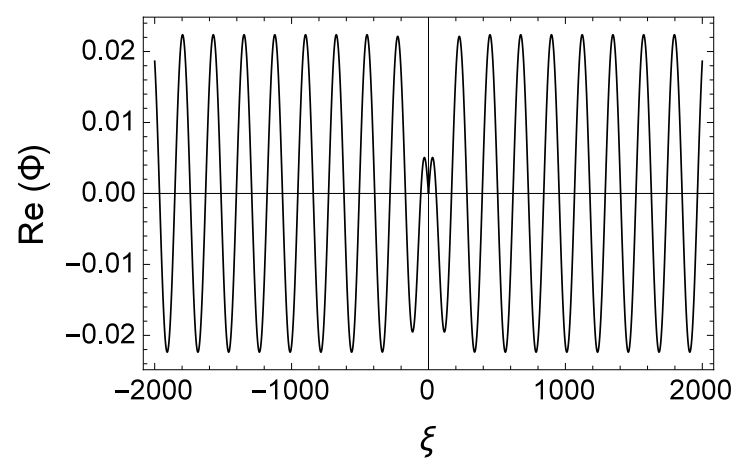

Fig. 11. The variation of $\operatorname{Re}(\Phi)$ with $\xi$ for dark envelope solitons; along with $k=0.2, \tau=0, \psi_{0}=0.0005, U=0.3, \Omega_{0}=0.4$, $a=1.5, b=0.08, q=1.3, \alpha=0.3, \delta=0.4, \mu_{i}=0.6, \sigma_{1}=0.0001, \sigma_{2}=0.001$, and $\omega_{f}$.

1. In the fast mode, both dust species oscillate in same phase with electrons and ions. On the other hand, in the slow mode one of the dust species oscillate in opposite phase with electrons, ions, and also another dust species.

2. For both $\omega_{f}$ and $\omega_{s}$, there is a stable/unstable region occurred for DAWs. DAWs are modulationally stable (unstable) for long (short) wavelength.

3. The maximum value of the $\Gamma_{g}$ increases with the decrease of $n_{i 0}$ for fixed value of $Z_{1}$ and $n_{10}\left(\right.$ via $\left.\mu_{i}=n_{i 0} / Z_{1} n_{10}\right)$. The growth rate increases with the $\beta$.

4. As we increase the value of $q$ in sub-extensive (super-extensive) limit of the $q$, the amplitude and width of the rogue waves decrease (increase).

The findings of our present investigation, which is useful to understand the nonlinear phenomena (viz. rogue waves and envelope solitons) in space DP (viz. ionosphere and mesosphere [3]) and laboratories plasmas (viz. high intensity laser irradiation and hot cathode discharge, etc. [3]) where $q$-distributed electrons and non-thermal ions as well as opposite polarity charged massive dust components are simultaneously co-exist.

\section{Acknowledgement}

M. H. Rahman is grateful to the Bangladesh Ministry of Science and Technology for awarding the National Science and Technology (NST) Fellowship.

\section{References}

1. D.A. Mendis, M. Rosenberg, Annu. Rev. Astron. Astrophys. 32, 419 (1994).

2. P.K. Shukla, Phys. Plasmas 8, 1791 (2001).

3. P.K. Shukla, A.A. Mamun, Introduction to Dusty Plasma Physics (Institute of Physics, Bristol, 2002).

4. F. Verheest, Waves in Dusty Plasmas (Kluwer Academic, Dordrecht, 2000).

5. N.A. Chowdhury, A. Mannan, A.A. Mamun, Phys. Plasmas 24, 113701 (2017).

6. S. Tasnim, A. Mannan, A. A. Mamun, Astrophys. Space Sci. 337, 261 (2012).

7. P.K. Shukla, V.P. Silin, Phys. Scr. 45, 508 (1992).

8. R. Bharuthram, P.K. Shukla, Planet. Space Sci. 40, 973 (1992).

9. A.A. Mamun, P.K. Shukla, Geophys. Res. Lett. 29, 1870 (2002).

10. V.W. Chow, D.A. Mendis, M. Rosenberg, J. Geophys. Res. 98, 19065 (1993).

11. V.W. Chow, D.A. Mendis, M. Rosenberg, IEEE Trans. Plasma Sci. 22, 179 (1994).

12. A. Renyi, Acta Math. Acad. Sci. Hung. 6, 285 (1995).

13. C. Tsallis, J. Stat. Phys. 52, 479 (1988).

14. N.A. Chowdhury, A. Mannan, M.M. Hasan, A.A. Mamun, Chaos 27, 093105 (2017).

15. M. Tribeche, A. Merriche, Phys. Plasmas. 18, 034502 (2011).

16. R.A. Cairns, A.A. Mamun, R. Bingham, R. Boström, R.O. Dendy, C.M.C. Nairn, P.K. Shukla, Geophys. res. lett. 22, 2709 (1995).

17. A.A. Mamun, R.A. Cairns, P.K. Shukla, Phys. Plasmas 3, 2610 (1996).

18. A.A. Mamun, Phys. Rev. E 55, 1852 (1997).

19. C. Kharif, E. Pelinovsky, A. Slunyaev, Rogue waves in the Ocean (Springer-Verlag, Berlin, 2009).

20. Z. Yan, Commun. Theor. Phys. 54, 947 (2010). 
21. A.N. Ganshin, V.B. Efimov, G.V. Kolmakov, L.P. Mezhov-Deglin, P.V.E. McClintock, Phys. Rev. Lett. 101, 065303 (2008).

22. W.M. Moslem, P.K. Shukla, B. Eliasson, Europhys. Lett. 96, 25002 (2011).

23. F. Sayed, A.A. Mamun, Phys. Plasmas 14, 014501 (2007).

24. W.F. El-Taibany, Phys. Plasmas 20, 093701 (2013).

25. A.S. Bains, M. Tribeche, C.S. Ng, Astrophys. Space Sci. 343, 621 (2013).

26. A.P. Misra, A.R. Chowdhury, Phys. Plasmas 39, 49 (2006).

27. S.K. Zaghbeer, H.H. Salah, N.H. Sheta, E.K. El-Shewy, A. Elgarayh, Astrophys. Space Sci. 353, 493 (2014).

28. W.M. Moslem, R. Sabry, S.K. El-Labany, P.K. Shukla, Phys. Rev. E 84, 066402 (2011).

29. S. Sultana, S. Islam, A.A. Mamun, Astrophys. Space Sci. 351, 581 (2014).

30. N.A. Chowdhury, M.M. Hasan, A. Mannan, A.A. Mamun, Vacuum 147, 31 (2018).

31. S. Sultana, I. Kourakis, Plasma Phys. Control. Fusion 53, 045003 (2011).

32. I. Kourakis, P.K. Shukla, Nonlinear Proc. Geophys. 12, 407 (2005).

33. R. Fedele, H. Schamel, Eur. Phys. J. B 27, 313 (2002) .

34. R. Fedele, Phys. Scr. 65, 502 (2002).

35. N. Akhmediev, A. Ankiewicz, J.M. Soto-Crespo, Phys. Rev. E 80, 026601 (2009).

36. A. Anikiewicz, N. Devine, N. Akhmediev, Phys. Lett. A 373, 3997 (2009). 